\title{
Identification of the key genes implicated in the transformation of OLP to OSCC using RNA-sequencing
}

\author{
QIAOZHEN YANG ${ }^{1 *}$, BIN GUO $^{2 *}$, HONGYING SUN $^{1}$, JIE ZHANG $^{1}$, SHANGFENG LIU $^{1}$, \\ SAIYIN HEXIGE ${ }^{2}$, XUEDI YU ${ }^{1}$ and XIAXIA WANG ${ }^{1}$ \\ ${ }^{1}$ Department of Stomatology, Huashan Hospital, Fudan University, Shanghai 200040; ${ }^{2}$ State Key Laboratory of \\ Genetic Engineering, Institute of Genetics, School of Life Sciences, Fudan University, Shanghai 200433, P.R. China
}

Received August 15, 2016; Accepted January 2, 2017

DOI: $10.3892 /$ or.2017.5487

\begin{abstract}
Oral lichen planus (OLP) is a chronic inflammatory disease that may transform to oral squamous cell carcinoma (OSCC), while its carcinogenesis mechanisms are not entirely clear. This study was designed to identify the important genes involved in the malignant transformation of OLP to OSCC. After RNA-sequencing, the differently expressed genes (DEGs) in OLP vs. normal and OSCC vs. normal groups, respectively, were identified by limma package in $\mathrm{R}$ language, and then clustering analysis were conducted by Pheatmap package in $\mathrm{R}$ language. Weighed gene co-expression network analysis (WGCNA) was performed for the DEGs to screen disease-associated modules. Using Cytoscape software, co-expression networks were constructed for the genes involved in the modules. Enrichment analysis was conducted for the genes involved in the co-expression networks using GOstat package in $\mathrm{R}$ language. Finally, quantitative real-time PCR (qRT-PCR) experiments were conducted to validate the key genes. There were, respectively, 223 and 548 DEGs in OLP vs. normal and OSCC vs. normal groups. WGCNA identified the blue modules for the DEGs in the two groups as disease-associated modules. Moreover, 19 common DEGs (including upregulated BCL9L, PER2 and TSPAN33, and downregulated GMPS and HES1) associated with both OLP and OSCC were identified. In the co-expression networks, BCL9L, HES1, PER2 and TSPAN33 might function in OLP via interactions (such as BCL9L-TSPAN33 and HES1-PER2). qRT-PCR analysis showed that BCL9L, PER2 and TSPAN33 were significantly upregulated, and GMP and HESI were
\end{abstract}

Correspondence to: Dr Hongying Sun, Department of Stomatology, Huashan Hospital, Fudan University, 12 Middle Wulumuqi Road, Shanghai 200040, P.R. China

E-mail: sunhongying@fudan.edu.cn

*Contributed equally

Keywords: oral lichen planus, oral squamous cell carcinoma, malignant transformation, RNA-sequencing, weighted gene co-expression network analysis downregulated. These findings indicated that BCL9L, GMPS, HES1, PER 2 and TSPAN33 affected the transformation of OLP to OSCC.

\section{Introduction}

Oral lichen planus (OLP) is a common chronic inflammatory disease of the oral mucosa, which is classified into oral potential malignant disorders (OPMDs) by World Health Organization (WHO) (1). OPMD is the general name of diseases occurring in oral mucosa and with cancerous potential (2). The incidence of OLP is $0.1-4 \%$ (3), and its canceration rate is close to $1 \%$ (4). The carcinogenesis mechanisms of OLP have been explored by many studies, but it is still not clear. Oral squamous cell carcinoma (OSCC) occurs in the mucosa of the oropharynx and oral cavity (5). In the United States, OSCC ranks 14th among cancers in women and 8th among cancers in men (6). OSCC is reported to take up $>90 \%$ of all oral cancers (7), and its incidence is increasing especially among white women (8). Previous studies report that OLP may transform to OSCC, though the incidence is low and the carcinogenesis mechanisms are not known $(9,10)$. Thus, investigating the mechanisms of malignant transformation of OLP to OSCC is important for further decreasing the incidence.

In recent years, malignant transformation of OLP has been widely studied. The matrix metalloproteinase-tissue inhibitor of matrix metalloproteinase (MMP-TIMP) imbalance may affect cancerization of OLP, additionally, MMP-9, MMP-2, and membrane-type $1 \mathrm{MMP}(M T 1-M M P)$ may serve as promising prognostic markers for malignant transformation of OLP $(11,12)$. The expression levels of ATP-binding cassette, G2 subfamily $(A B C G 2)$ and podoplanin are significantly related to malignant potential of OLP, suggesting that $A B C G 2$ and podoplanin may be used for assessing the risk of malignant transformation in OLP patients (13). Rhodus et al demonstrated that the abnormal expression levels of nuclear factor $\kappa \mathrm{B}$ $(\mathrm{NF}-\kappa \mathrm{B})$-dependent cytokines (such as interleukin-1 $\alpha$, IL-1 $\alpha$; and tumor necrosis factor- $\alpha, \mathrm{TNF}-\alpha$ ) in general, unstimulated saliva may reflect the malignant potential of OLP $(14,15)$. Overexpression of cyclin-dependent kinase $4(\mathrm{cdk} 4)$ and p16 indicate the cell arrest and hyperproliferative state of epithelial cells in OLP, and offer evidence for the malignant transforma- 
tion risk of OLP (16). However, the malignant transformation of OLP has not been comprehensively investigated.

RNA-sequencing is a powerful tool for expression profiling, genome annotation, and transcript discovery, which has been widely utilized in biology fields involving development, gene regulation and disease (17). In this study, the high-throughput sequencing data of OLP, OSCC and normal oral mucosa were obtained by RNA-sequencing technique. Followed by the differently expressed genes (DEGs) in OLP vs. normal and OSCC vs. normal comparison groups were identified. Subsequently, weighted gene co-expression network analysis (WGCNA) was conducted for the DEGs to screen disease-associated modules. Moreover, co-expression networks were constructed for the genes involved in the disease-associated modules. In addition, functional enrichment analysis was conducted for the genes involved in the co-expression networks. Finally, the identified key genes were further confirmed.

\section{Materials and methods}

Sample source. A total of 3 OLP samples, 3 OSCC samples, and 3 normal samples were isolated from buccal mucosa of patients from Huashan Hospital. The isolated samples were washed by phosphate buffer and then stored at $-80^{\circ} \mathrm{C}$ for following experiments. The patients had not received immune stimulants (including corticosteroids) within 3 months, and were without systemic diseases such as diabetes and immunodeficiency. Based on the diagnostic criteria of World Trade Organization (WTO), the patients were diagnosed by tissue biopsy. All patients gave their informed consent, and this study was approved by the ethics review committee of Huashan Hospital of Fudan University.

RNA extraction and library construction. Total RNA of the samples were extracted by the TRIzol total RNA extraction kit (Invitrogen, Shanghai, China) according to the manufacturer's manual. Subsequently, the integrity and purity of RNA were detected by $2 \%$ Agarose Gel Electrophoresis and spectrophotometer (Merinton, Beijing, China), respectively. The cDNA library was prepared using NEBNext ${ }^{\circledR}$ Ultra $^{\mathrm{TM}}$ RNA Library Prep kit for Illumina ${ }^{\circledR}$ (New England Biolabs, Ipswich, MA, USA). Firstly, the isolated mRNAs were fractured into short fragments ( $200 \mathrm{nt})$ through heating. Secondly, the first- and second-strand of cDNA were synthesized and then modified. Followed by PCR amplification. The cDNA library was sequenced on Illumina Hiseq 2500 v4 100PE (Illumina Inc., San Diego, CA, USA) to obtained the raw data.

Sequence alignment and DEG screening. Reads with adaptor sequences, and with $>50 \%$ low quality bases and/or with $>10 \%$ unknown nucleotides were defined as low quality sequences. Using NGSQC Toolkit (http://www.nipgr.res.in/ngsqctoolkit. html) (18), the raw data were pre-processed by filtering out the low quality sequences. Afterwards, the high quality sequences were aligned to human genome (version hg19) using tophat2 software (http://ccb.jhu.edu/software/tophat/index.shtml) (19), with default parameters. Furthermore, the DEGs in OLP vs. normal and OSCC vs. normal comparison groups, respectively, were screened by the limma package (http://www.bioconductor.org/packages/release/bioc/html/limma.html) $(20,21)$ in
Table I. The primers used for quantitative real-time PCR (qRT-PCR) experiments.

\begin{tabular}{ll}
\hline Primer name & \multicolumn{1}{c}{ Primer sequence (5'-3') } \\
\hline BCL9L & F: CACAATGCCATCAAGACCATC \\
& R: AGTTCAGGTGCATCTGGCTG \\
GMPS & F: CATAGACCGAAGAGTGAGGGAAC \\
& R: GAACAGGCTTGCCAATAGTGAATA \\
HES1 & F: CAGCGAGTGCATGAACGAGGTGA \\
& R: AGGTGCCGCTGTTGCTGGTGTAGA \\
PER2 & F: TCCAGATACCTTTAGCCTGATGA \\
& R: TTTGTGTGTGTCCACTTTCGA \\
TSPAN33 & F: GGCAAGCCTCATAAACGAAC \\
& R: CCTTCTGCCCATCTGGAGTT \\
GAPDH & F: TGACAACTTTGGTATCGTGGAAGG \\
& R: AGGCAGGGATGATGTTCTGGAGAG
\end{tabular}

$\mathrm{R}$ language. The thresholds for screening DEGs were $\log _{2}$ fold change $(\mathrm{FC}) \mid>0.5$ and $\mathrm{P}$-value $<0.05$.

Euclidean distance, which can be calculated by the Pythagorean formula, refers to the true distance between two points (22). The expression values of the DEGs in each sample were extracted. By the Pheatmap package (http://cran.r-project. org/web/packages/pheatmap/index.html) (23) in R language, bidirectional hierarchical clustering analysis $(24,25)$ were performed to cluster gene expression values and samples based on the Euclidean distance (22). After that, genes with close expression were clustered together, and genes with sample specificity can be identified.

Screening disease-associated module using WGCNA. WGCNA is a typical systems biology method for constructing gene co-expression network, which is based on highthroughput gene expression data (26,27). Firstly, the Pearson's correlation matrices were calculated for each gene pair, and the correlation coefficient $\mathrm{S}_{\mathrm{mn}}|\operatorname{cor}(\mathrm{m}, \mathrm{n})|$ was defined for $m$ and $n$ gene pairs. Secondly, the Pearson's correlation matrices were converted into adjacent matrices by an adjacent function $a_{m n}$ power $\left(S_{m n}, \beta\right)$. Then, the weighting coefficient $\beta$ was determined based on the principle of scale-free network. The weighting coefficient $\beta$, which was the correlation coefficient between $\log _{2} \mathrm{k}$ and $\log _{2} \mathrm{p}(\mathrm{k})$ ( $\mathrm{k}$ represented the number of connected nodes, and $\mathrm{p}$ stood for appearing probability of nodes) should be no less than 0.9. Subsequently, the adjacent matrices were transformed into topology matrices using $\Omega=$ $\mathrm{W}_{\mathrm{mn}}=\left(\mathrm{l}_{\mathrm{mn}}+\mathrm{a}_{\mathrm{mn}}\right) /\left(\min \left\{\mathrm{k}_{\mathrm{m}}, \mathrm{k}_{\mathrm{n}}\right\}+1-\mathrm{a}_{\mathrm{mn}}\right)$. The $\mathrm{l}_{\mathrm{mn}}$ represented the total area of correlation coefficients of the nodes linked with both gene $m$ and gene $n$. The $k_{m}$ indicated the sum of correlation coefficients of the nodes linked with gene $\mathrm{m} . \mathrm{W}_{\mathrm{mn}}$ was equal to 0 , when gene $m$ did not connect with gene $n$, and no genes linked with both gene $\mathrm{m}$ and gene $\mathrm{n}$. The dissimilarity degree of a node, which was the basis for constructing the network, was defined as $d_{m n}=1-W_{m n}$. WGCNA for the DEGs was conducted as described previously $(26,27)$, and gene modules were identified by hybrid dynamic shear tree $(28,29)$. Besides, T-test was applied to calculate the 
Table II. The quality control results and the comparison results of the raw RNA-sequencing data.

A, Quality control results

\begin{tabular}{lrcccccc}
\hline Sample & Raw reads & Raw bases & Trim reads & Trim bases & $\begin{array}{c}\text { Average } \\
\text { length }\end{array}$ & $\begin{array}{c}\text { Trim reads } \\
(\%)\end{array}$ & $\begin{array}{c}\text { Trim bases } \\
(\%)\end{array}$ \\
\hline OLP & 87356096 & 8822965696 & 77746400 & 7331570314 & 94.30109065 & 0.889993985 & 0.830964391 \\
OSCC & 79835924 & 8063428324 & 66754732 & 6087961474 & 91.19895012 & 0.83614905 & 0.755009064 \\
Normal & 100217370 & 10121954370 & 81959072 & 7494718324 & 91.44464598 & 0.81781304 & 0.740441821 \\
\hline
\end{tabular}

$\mathrm{B}$, Comparison results

\begin{tabular}{lccccccr}
\hline Sample & Total & Total & $\begin{array}{c}\text { Mapped } \\
\text { ratio } \\
(\%)\end{array}$ & $\begin{array}{c}\text { Multiple } \\
\text { mapped }\end{array}$ & $\begin{array}{c}\text { Unique } \\
\text { mapped }\end{array}$ & $\begin{array}{c}\text { Reads } \\
\text { mapping to } \\
\text { positive-sense }\end{array}$ & $\begin{array}{c}\text { Reads } \\
\text { mapping to } \\
\text { antisense strand }\end{array}$ \\
\hline OLP & 77746400 & 63674055 & 81.90 & 2979371 & 60694684 & 30207215 & 30487469 \\
OSCC & 66754732 & 48292145 & 72.30 & 3068746 & 45223399 & 22535431 & 22687968 \\
pair \\
Normal & 81959072 & 53529855 & 65.30 & 3492342 & 50037513 & 24873837 & 25163676 \\
\hline
\end{tabular}

OLP, oral lichen planus; OSCC, oral squamous cell carcinoma.

significant P-value of each gene between different groups, and the mediated P-value $(\lg \mathrm{P})$ was defined as the gene significance (GS) of each gene. The module significance (MS) was considered as the mean GS of the genes included in the module. Among the identified modules, the one with the highest MS value was selected as disease-associated module.

Construction of co-expression network for genes involved in the disease-associated module. The correlation coefficients of genes involved in the OLP-associated module and OSCC-associated module separately were extracted from the modules. Then, the co-expression networks were visualized by Cytoscape software (http://www.cytoscape.org/) (30). Besides, the genes associated with both OLP and OSCC were identified by comparing the genes involved in OLP-associated module and OSCC-associated module. Moreover, the genes associated with both OLP and OSCC were mapped to the co-expression networks.

Functional enrichment analysis. GOstats (available at: http:// bioconductor.org) is usually utilized to perform functional enrichment analysis and test gene ontology (GO) terms for genes in a specific gene list (31). GO can be used to analyze the biological process, molecular function and cellular component involved gene products (32). Using the GOstat package (31) in $\mathrm{R}$ language, $\mathrm{GO}$ enrichment analysis was conducted for the disease-associated genes involved in the co-expression network. The terms with P-value $<0.05$ were taken as statistically significant.

$q R T-P C R$ analysis. After the primer sequences for qRT-PCR amplification were designed using Primer Premier 6.0 software (Premier Software Inc., Cherry Hill, NJ, USA) (Table I), they were synthesized by Sangon Biotech Co., Ltd (Shanghai,
China). The expression of critical genes in OLP, OSCC and normal samples were measured by SYBR Green master mix kit (Applied Biosystems, Foster City, CA), respectively. The $20 \mu \mathrm{l}$ reaction system was composed of the following reagents: $10 \mu 1$ SYBR Premix Ex Taq (2X), $1 \mu$ l forward primer $(10 \mu \mathrm{M})$, and $1 \mu \mathrm{l}$ reverse primer $(10 \mu \mathrm{M})$, and $8 \mu \mathrm{l}$ cDNA template (being diluted by $\mathrm{ddH}_{2} \mathrm{O}$ to keep a certain concentration). Then, the mixture reacted under the following conditions: $50^{\circ} \mathrm{C}$ for $3 \mathrm{~min} ; 95^{\circ} \mathrm{C}$ for $3 \mathrm{~min} ; 95^{\circ} \mathrm{C}$ for $10 \mathrm{sec}$ and $60^{\circ} \mathrm{C}$ for $30 \mathrm{sec}$ for 40 cycles; melt curve $60-95^{\circ} \mathrm{C}$ : increment $0.5^{\circ} \mathrm{C}$ for $10 \mathrm{sec}$ plate read. All samples had three repeats, with GAPDH as the reference gene.

Statistical analysis. Using the $2^{-\Delta \Delta C t}$ method (33), the gene expression values were calculated. All results are presented as mean \pm SEM (standard error of mean). SPSS 22.0 (SPSS Inc., Chicago, IL, USA) and Graphpad prism 5 (Graphpad Software, San Diego, CA, USA) software was used also for statistic analysis and drawing pictures, with the $\mathrm{P}<0.05$ as the screening criteria for significant difference.

\section{Results}

DEG screening and bidirectional hierarchical clustering. The raw data were preprocessed, and the quality control results and the comparison results of the raw RNA-sequencing data, respectively, are shown in Table IIA and Table IIB. Followed by a total of 223 (including 74 up- and 149 down-regulated genes) and 548 (including 80 up- and 468 down-regulated genes) DEGs separately were identified in OLP vs. normal and OSCC vs. normal comparison groups. Subsequently, the expression values of the DEGs in each sample were extracted and bidirectional hierarchical clustering analysis was carried out. The dendrogram of clustering analysis showed that the DEGs could separate the OLP or OSCC samples from normal 

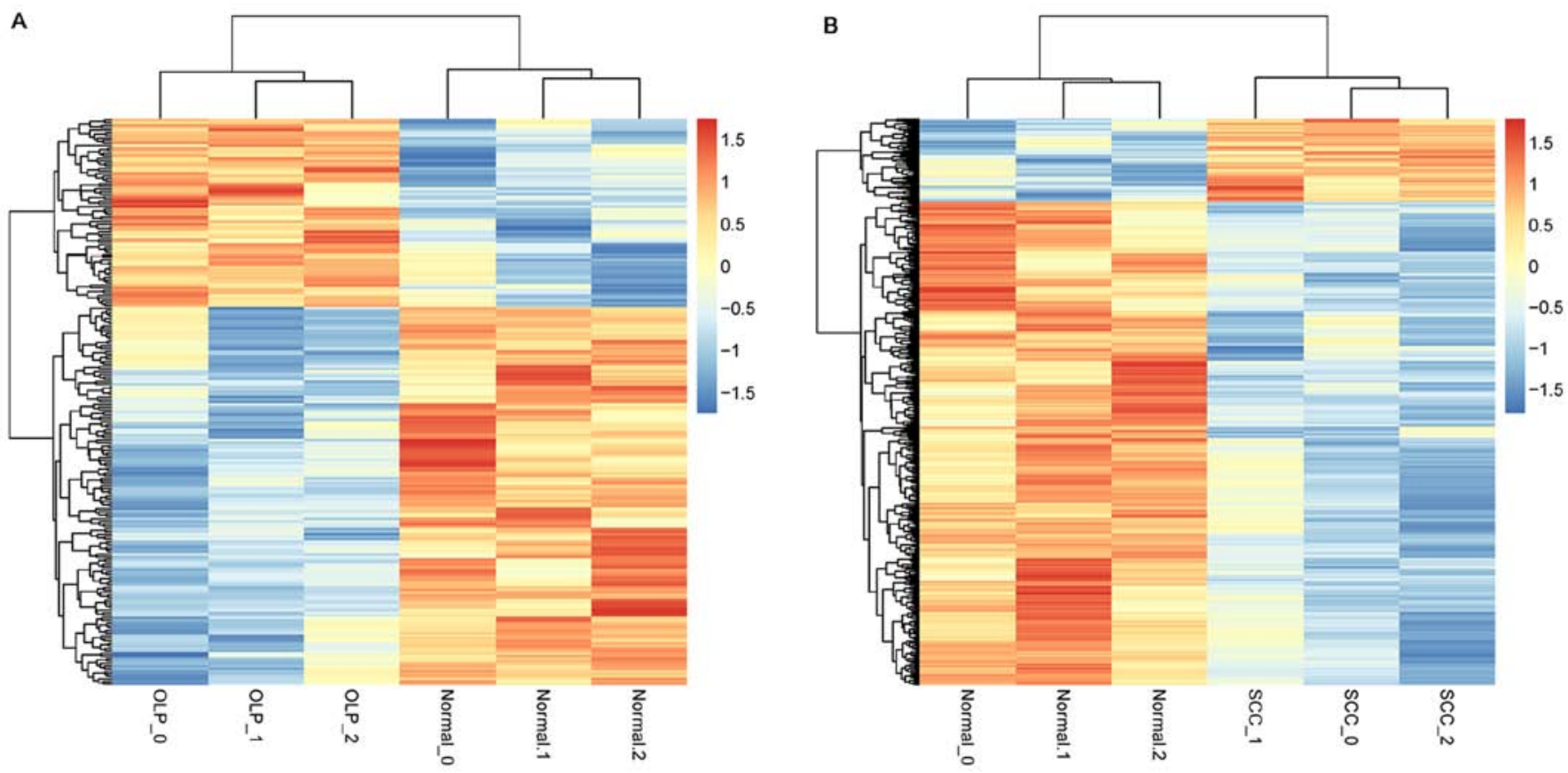

Figure 1. The dendrogram of clustering analysis for the differentially expressed genes in OLP vs. normal (A) and OSCC vs. normal (B) comparison groups. Red and blue indicate higher and lower expression values, respectively.

A

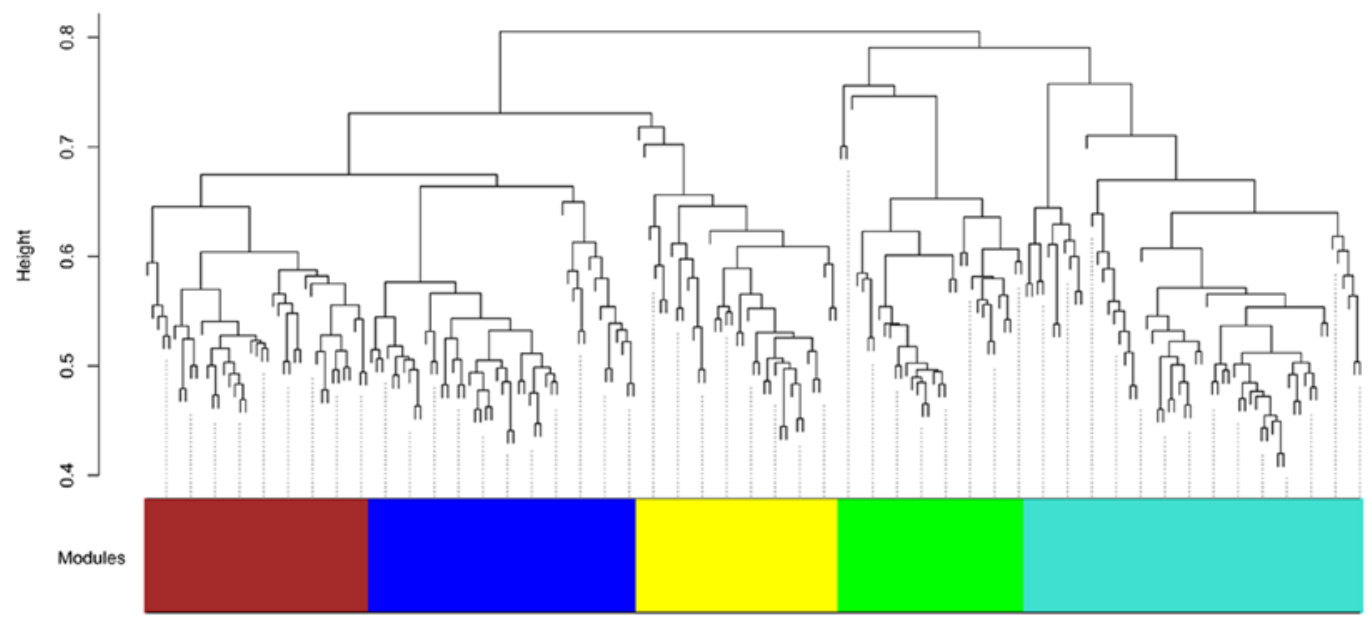

B

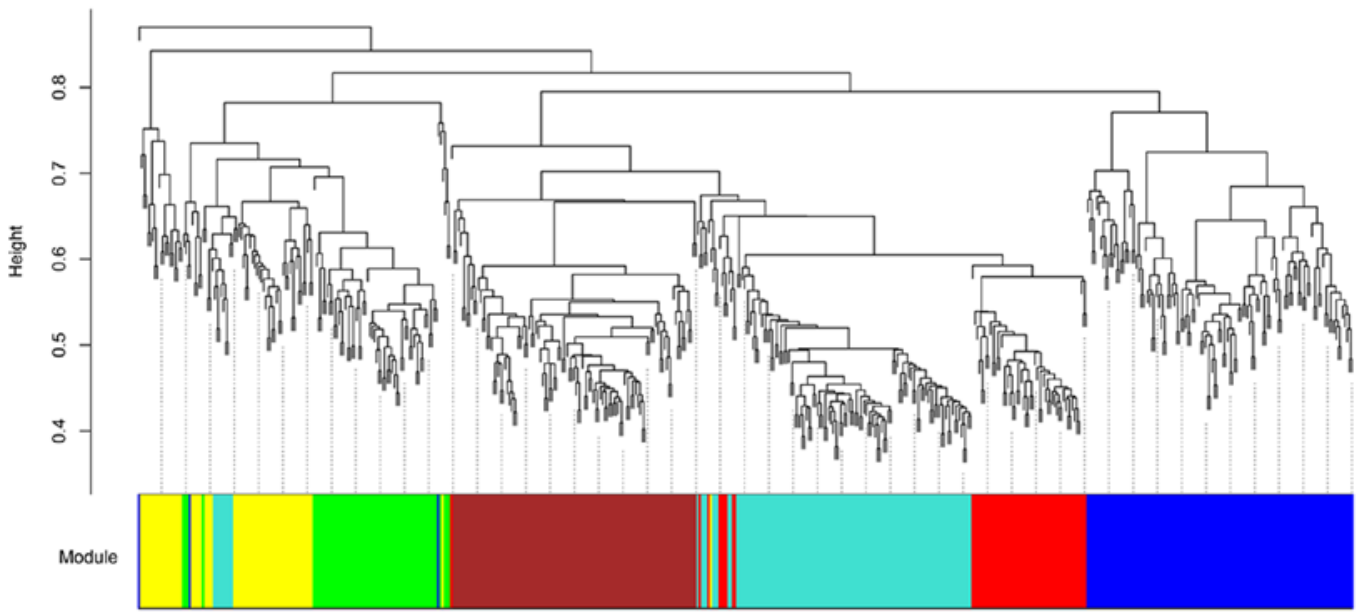

Figure 2. Modules identified for the differentially expressed genes in OLP vs. normal (A) and OSCC vs. normal (B) comparison groups. Horizontal ordinate represents modules in different colors. Vertical ordinate is the height of clustering tree. 

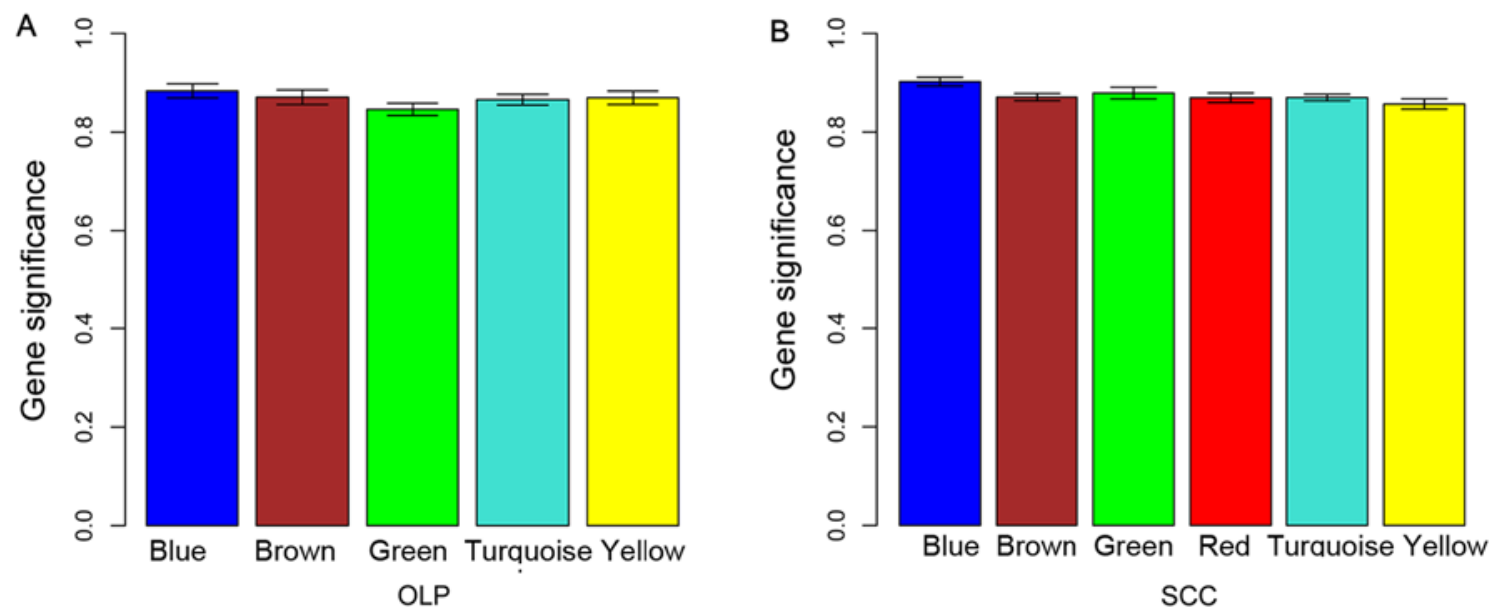

Figure 3. Identification of OLP- (A) and OSCC-associated (B) modules based on the MS values. Horizontal ordinate represents modules in different colors Vertical ordinate is the overall correlation coefficient between genes in each module and disease state.

Table III. The common differentially expressed genes (DEGs) between OLP vs. normal and OSCC vs. normal comparison groups.

\begin{tabular}{llllll}
\hline & \multicolumn{2}{c}{ OLP } & & \multicolumn{2}{c}{ SCC } \\
\cline { 2 - 3 } \cline { 6 - 6 } Gene & logFC & P-value & & logFC & P-value \\
\hline BCL9L & 0.866268 & 0.027103 & & 0.87862 & 0.011379 \\
C21orf62 & -0.87082 & 0.034486 & & -0.87537 & 0.025741 \\
CHGA & -0.87469 & 0.030446 & & -0.89057 & 0.009506 \\
DPY19L2P4 & -0.84725 & 0.003033 & & -0.87498 & 0.006929 \\
FAM228B & -0.89934 & 0.048295 & & -0.91674 & 0.025529 \\
GMPS & -0.87338 & 0.001723 & & -0.86493 & 0.008399 \\
HES1 & -0.88283 & 0.010514 & & -0.87028 & 0.004662 \\
OIP5-AS1 & 0.880789 & 0.000374 & & 0.881847 & 0.000254 \\
PER2 & 0.882885 & 0.011876 & & 0.886616 & 0.004227 \\
SMC6 & -0.8837 & 0.016755 & & -0.88647 & 0.048216 \\
SNX29P1 & -0.92343 & 0.019689 & & -0.94756 & 0.01687 \\
SPAC1L & -0.86816 & 0.020417 & & -0.87373 & 0.015081 \\
TERF2 & -0.87686 & 0.038943 & & -0.8964 & 0.012173 \\
TMEM121 & -0.90655 & 0.045068 & & -0.87188 & 0.033126 \\
TSPAN33 & 0.843168 & 0.005186 & & 0.835443 & 0.046788 \\
ZNF347 & -0.8933 & 0.0388 & & -0.89562 & 0.023093 \\
ZNF439 & -0.9103 & 0.019051 & & -0.90591 & 0.014761 \\
ZNF669 & -0.87 & 0.018227 & & -0.86737 & 0.01926 \\
ZNF70 & -0.86138 & 0.007926 & -0.8725 & 0.00475 \\
\hline
\end{tabular}

samples completely, indicating that the expression differences of the DEGs were significant (Fig. 1).

Screening disease-associated module using WGCNA. Based on hybrid dynamic shear tree, modules for the DEGs in the two comparison groups were identified and exhibited by different colors (Fig. 2). According to the MS value of modules, the blue modules for the DEGs in OLP vs. normal $(\mathrm{MS}=0.88)$ and in OSCC vs. normal $(\mathrm{MS}=0.90)$ comparison groups were selected as disease-associated modules for they had the highest MS values (Fig. 3)

Construction of co-expression network for genes involved in the disease-associated module. There were 49 and 123 DEGs, respectively, in the blue modules for the DEGs in OLP vs. normal and in OSCC vs. normal comparison groups. The correlation coefficients of genes involved in the blue modules were extracted, and the co-expression networks were visualized. Through comparing the genes involved in the blue modules, a total of 19 common DEGs (including 4 upregulated genes and 15 downregulated genes) associated with both OLP and OSCC were identified and mapped to the co-expression networks (Table III), including upregulated B-cell CLL/lymphoma 9-like (BCL9L), period circadian clock 2 (PER2) and tetraspanin 33 (TSPAN33), as well as downregulated guanine monphosphate synthase (GMPS) and Hes family bHLH transcription factor 1 (HES1). The co-expression network for OLP vs. normal comparison group had 138 interactions and 41 nodes, and that for OSCC vs. normal comparison group had 757 interactions and 85 nodes (Fig. 4). Especially, BCL9L, HES1, PER2 and TSPAN33 might be involved in OLP through interactions in the co-expression networks for OLP vs. normal (such as BCL9L-TSPAN33) and OSCC vs. normal comparison groups (such as BCL9L-PER2, and HES1-PER2).

Functional enrichment analysis. GO enrichment analysis was performed for the DEGs involved in the co-expression networks for OLP vs. normal and OSCC vs. normal comparison groups, respectively. In total, 7 and 6 GO terms, respectively, were enriched for the DEGs involved in the co-expression networks for OLP vs. normal and OSCC vs. normal comparison groups (Fig. 5 and Table IV). Importantly, the functions enriched for the DEGs involved in the two co-expression networks both included transcription, regulation of transcription, regulation of transcription, DNA-dependent, and regulation of RNA metabolic process.

qRT-PCR analysis. Through qRT-PCR experiments, the expression levels of $B C L 9 L, G M P S, H E S 1, P E R 2$ and 
A
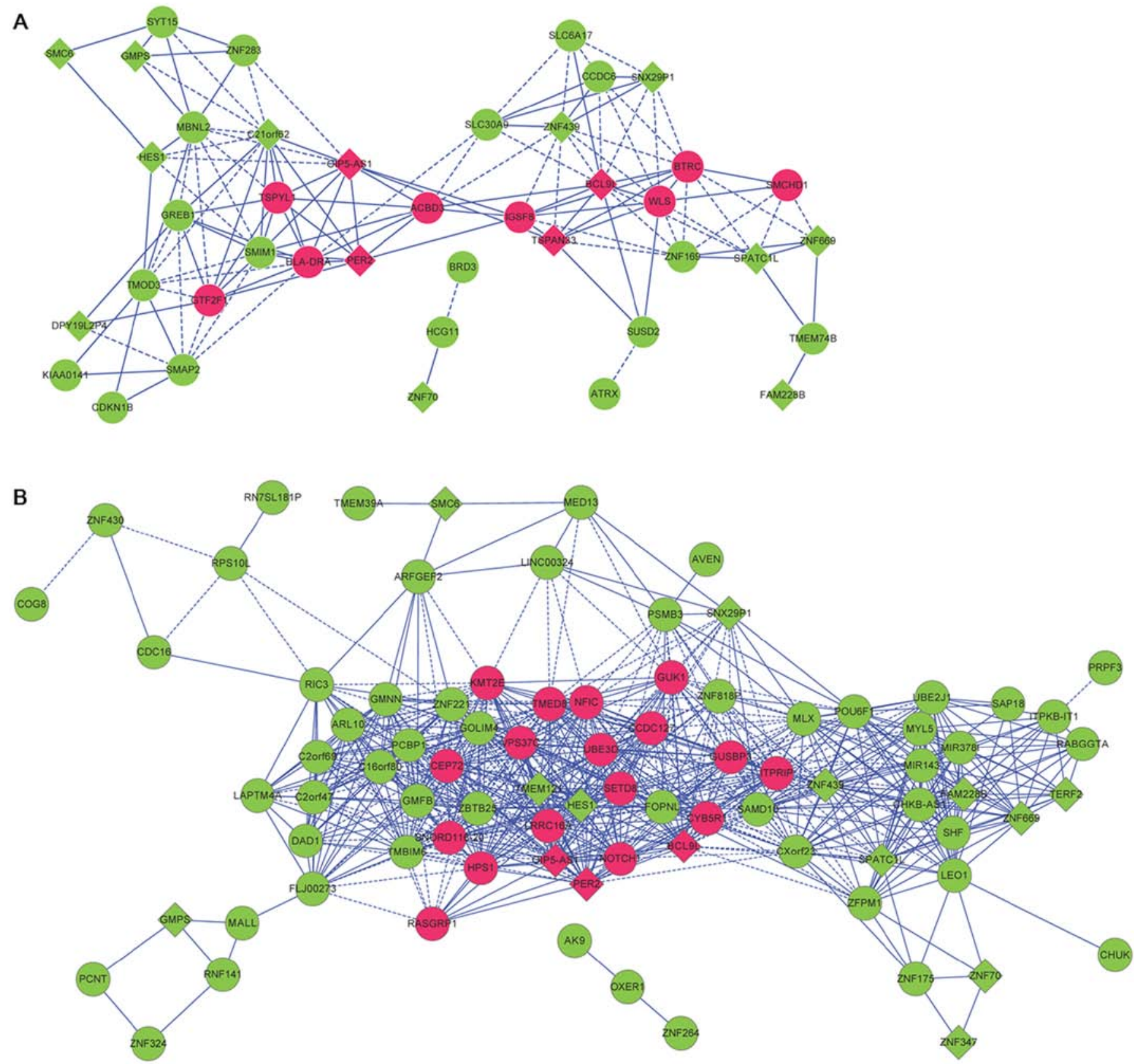

Figure 4. The co-expression networks for the differentially expressed genes (DEGs) in the blue modules. (A) The co-expression network for the DEGs in the blue module for OLP vs. normal comparison group. (B) The co-expression networks for the DEGs in the blue modules for OSCC vs. normal comparison groups. Red and green nodes represent upregulated and downregulated genes, respectively. Diamond nodes stood for the common DEGs between the OLP vs. normal and OSCC vs. normal comparison groups. Solid lines and dotted lines indicate positive correlations and negative correlations, respectively.

TSPAN33 in OLP, OSCC and normal samples were detected. Compared with normal samples, $B C L 9 L(\mathrm{P}<0.05$, Fig. 6A), PER2 (P<0.05, Fig. 6B) and TSPAN33 (P<0.05, Fig. 6C) were significantly upregulated in both OLP and OSCC samples. GMPS (P<0.05, Fig. 6D) in both OLP and OSCC samples, and HESI ( $\mathrm{P}<0.05$, Fig. 6E) in OSCC samples were significantly downregulated relative to normal samples.

\section{Discussion}

In OLP subepithelial infiltrate, cytokine networks and inflammatory cells may contribute to squamous tumorigenesis through affecting cell survival, proliferation, differentiation, growth and movement (34). Conway et al analyzed the transcriptomes of matched OSCC, oral dysplasia and normal oral mucosa samples, finding that IL36G and adherens junction components play potential roles in malignant transformation, and lincRNA RP11-351J23.1 acts in de-differentiation of OSCCs (35). Jakhesara et al investigated the aberrant transcriptional events in buccal mucosal cancer (BMC) through high throughput RNA-Seq analysis, and obtain 588 DEGs and 747 dysregulated isoforms that may be used for therapeutic intervention and biomarker evaluation (36). Tang et al compared the gene expression changes between a late stage and an early stage of tongue carcinogenesis, and reveal ALDH1A3, PTGS2 and KRT1 transcripts, as well as 
Table IV. The gene ontology (GO) terms enriched for the differentially expressed genes (DEGs) involved in the co-expression networks for OLP vs. normal and OSCC vs. normal comparison groups.

A, The functions enriched for the DEGs involved in the co-expression network for OLP vs. normal comparison group

\begin{tabular}{|c|c|c|c|}
\hline Description & P-value & Gene no. & Gene symbol \\
\hline $\begin{array}{l}\text { GO:0045449 regulation of } \\
\text { transcription }\end{array}$ & $1.78 \mathrm{E}-03$ & 12 & $\begin{array}{l}\text { ZNF169, ATRX, HES1, ZNF439, ZNF283, CDKN1B, } \\
\text { GTF2F1, PER2, BCL9L, ZNF669, ZNF70, SLC30A9 }\end{array}$ \\
\hline GO:0006350 transcription & $5.32 \mathrm{E}-03$ & 10 & $\begin{array}{l}\text { ZNF169, HES1, ZNF439, ZNF283, GTF2F1, PER2, } \\
\text { BCL9L, ZNF669, ZNF70, SLC30A9 }\end{array}$ \\
\hline $\begin{array}{l}\text { GO:0006355 regulation of } \\
\text { transcription, DNA-dependent }\end{array}$ & $6.78 \mathrm{E}-03$ & 9 & $\begin{array}{l}\text { ZNF169, ATRX, HES1, ZNF439, ZNF283, CDKN1B, } \\
\text { PER2, ZNF669, ZNF70 }\end{array}$ \\
\hline $\begin{array}{l}\text { GO:0051252 regulation of RNA } \\
\text { metabolic process }\end{array}$ & $7.76 \mathrm{E}-03$ & 9 & $\begin{array}{l}\text { ZNF169, ATRX, HES1, ZNF439, ZNF283, CDKN1B, } \\
\text { PER2, ZNF669, ZNF70 }\end{array}$ \\
\hline GO:0008270 zinc ion binding & $1.65 \mathrm{E}-02$ & 9 & $\begin{array}{l}\text { ZNF169, ATRX, SMAP2, ZNF439, ZNF283, ZNF669, } \\
\text { MBNL2, ZNF70, SLC30A9 }\end{array}$ \\
\hline GO:0003677 DNA binding & $1.74 \mathrm{E}-02$ & 9 & $\begin{array}{l}\text { ZNF169, ATRX, HES1, ZNF439, ZNF283, GTF2F1, } \\
\text { ZNF669, ZNF70, SLC30A9 }\end{array}$ \\
\hline $\begin{array}{l}\text { GO:0046914 transition metal } \\
\text { ion binding }\end{array}$ & $4.68 \mathrm{E}-02$ & 9 & $\begin{array}{l}\text { ZNF169, ATRX, SMAP2, ZNF439, ZNF283, ZNF669, } \\
\text { MBNL2, ZNF70, SLC30A9 }\end{array}$ \\
\hline
\end{tabular}

$\mathrm{B}$, The functions enriched for the DEGs involved in the co-expression network for OSCC vs. normal comparison group

\begin{tabular}{|c|c|c|c|}
\hline Description & P-value & Gene no. & Gene symbol \\
\hline GO:0006350 transcription & $3.41 \mathrm{E}-06$ & 23 & $\begin{array}{l}\text { POU6F1, ZNF430, ZNF264, ZNF818P, SAP18, } \\
\text { ZNF669, MED13, ZNF221, ZNF347, ZNF175, } \\
\text { ZBTB25, HES1, ZNF439, NOTCH1, ZNF324, MLX, } \\
\text { PER2, LEO1, BCL9L, SETD8, ZFPM1, NFIC, ZNF70 }\end{array}$ \\
\hline $\begin{array}{l}\text { GO:0045449 regulation of } \\
\text { transcription }\end{array}$ & $9.08 \mathrm{E}-06$ & 25 & $\begin{array}{l}\text { POU6F1, ZNF430, ZNF264, ZNF818P, SAP18, } \\
\text { ZNF669, MED13, ZNF221, ZNF347, ZNF175, } \\
\text { ZBTB25, HES1, ZNF439, NOTCH1, RNF141, } \\
\text { ZNF324, MLX, PER2, LEO1, BCL9L, SETD8, } \\
\text { ZFPM1, NFIC, ZNF70, TERF2 }\end{array}$ \\
\hline $\begin{array}{l}\text { GO:0006355 regulation of } \\
\text { transcription, DNA-dependent }\end{array}$ & $2.16 \mathrm{E}-04$ & 18 & $\begin{array}{l}\text { POU6F1, ZNF430, ZNF264, SAP18, ZNF669, MED13, } \\
\text { ZNF221, ZNF347, ZNF175, HES1, NOTCH1, ZNF439, } \\
\text { RNF141, ZNF324, MLX, PER2, NFIC, ZNF70 }\end{array}$ \\
\hline $\begin{array}{l}\text { GO:0051252 regulation of } \\
\text { RNA metabolic process }\end{array}$ & $2.83 \mathrm{E}-04$ & 18 & $\begin{array}{l}\text { POU6F1, ZNF430, ZNF264, SAP18, ZNF669, MED13, } \\
\text { ZNF221, ZNF347, ZNF175, HES1, NOTCH1, ZNF439, } \\
\text { RNF141, ZNF324, MLX, PER2, NFIC, ZNF70 }\end{array}$ \\
\hline $\begin{array}{l}\text { GO:0010605 negative regulation of } \\
\text { macromolecule metabolic process }\end{array}$ & $2.15 \mathrm{E}-02$ & 8 & $\begin{array}{l}\text { HES1, PSMB3, MLX, GMNN, SETD8, CDC16, NFIC, } \\
\text { TERF2 }\end{array}$ \\
\hline $\begin{array}{l}\text { GO:0051172 negative regulation of } \\
\text { nitrogen compound metabolic process }\end{array}$ & 4.83E-02 & 6 & HES1, MLX, GMNN, SETD8, NFIC,TERF2 \\
\hline
\end{tabular}

pathways of 'degradation of basement membrane and ECM pathways' and 'cell cycle progression' may function in early diagnosis and prevention of human tongue squamous cell carcinomas (37). Somoza-Martín et al analyzed the gene expression profile of OSCC, and identified 322 upregulated genes and 104 downregulated genes in tumoral tissue (38). However, these findings were different from our results. In this study, total 223 (including 74 up- and 149 down-regulated genes) and 548 (including 80 up- and 468 down-regulated genes) DEGs, respectively, were identified in OLP vs. normal and OSCC vs. normal comparison groups. The dendrogram of clustering analysis showed that the DEGs completely separated the OLP or OSCC samples from normal samples. Besides, the blue modules for the DEGs in OLP vs. normal 
A

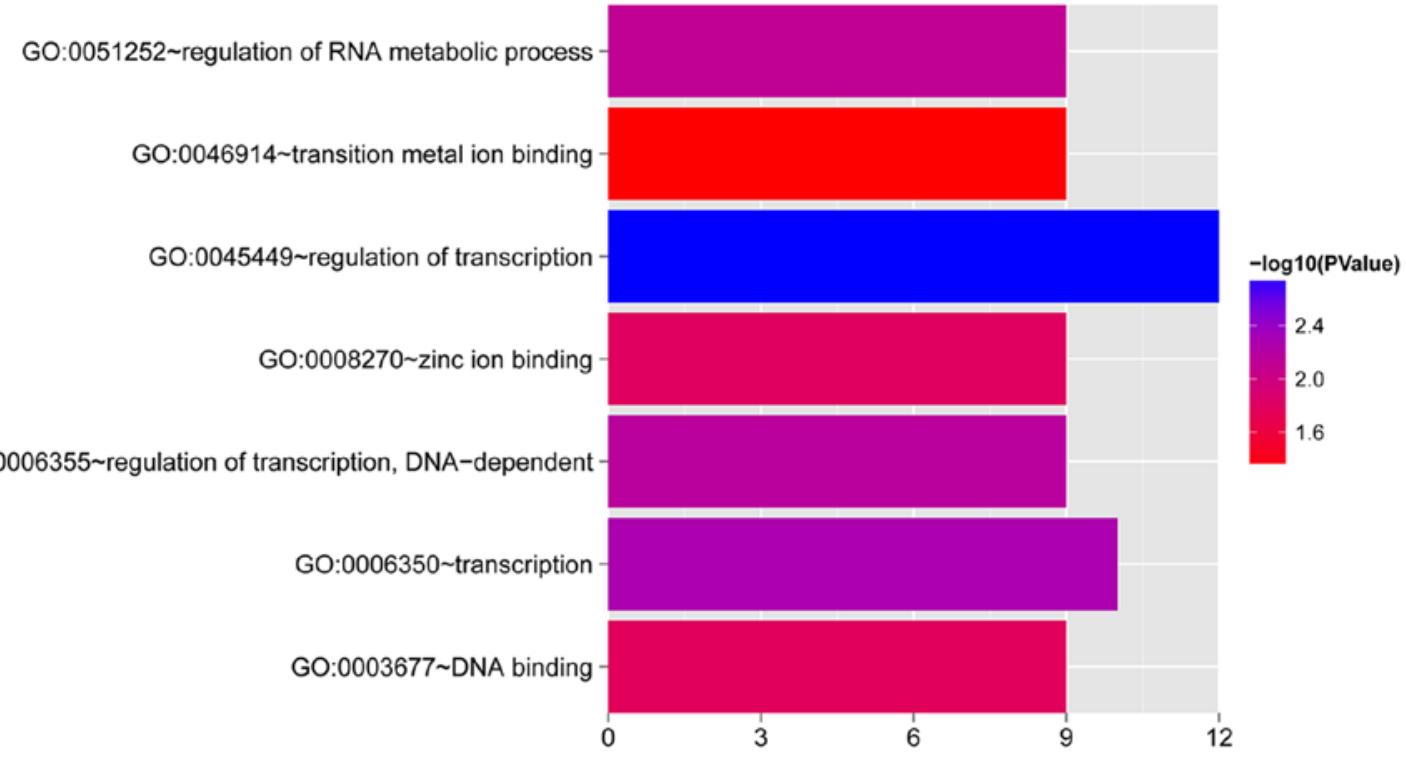

B

GO:0051252 regulation of RNA metabolic process

GO:0051172 negative regulation of nitrogen compound metabolic process

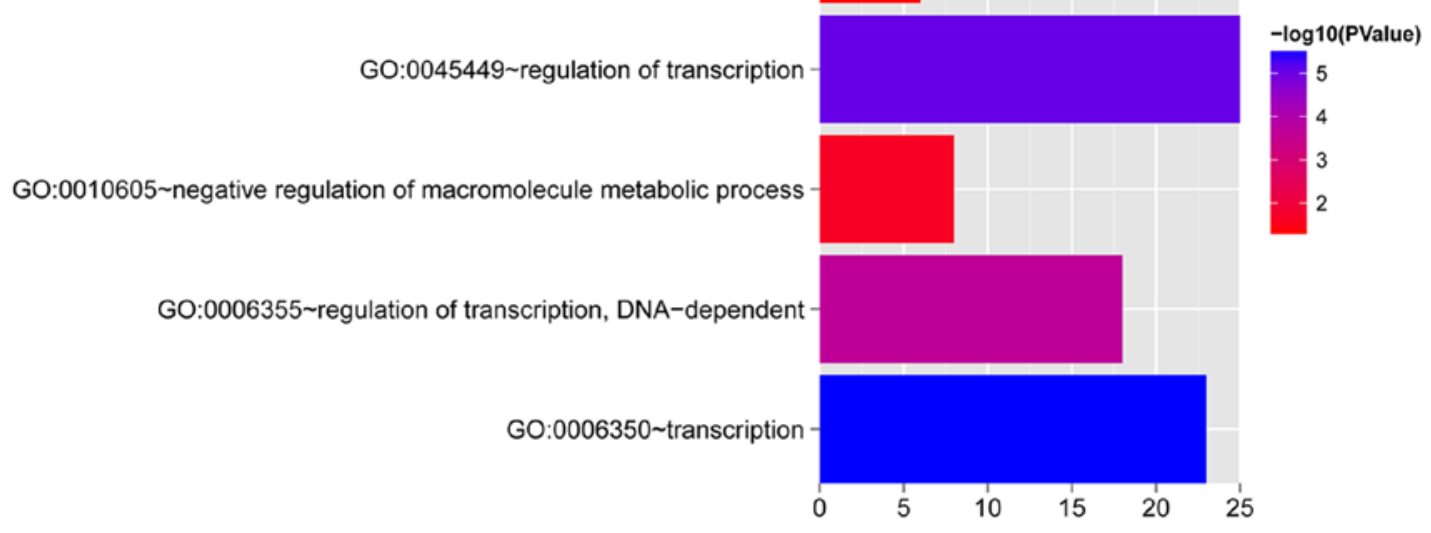

Figure 5. The functional terms enriched for the differentially expressed genes involved in the co-expression networks for OLP vs. normal (A) and OSCC vs. normal (B) comparison groups. The height of the column indicates the number of genes involved in the term. The color of the column represents the $-\lg (\mathrm{P}$-value $)$ value.

and in OSCC vs. normal comparison groups were diseaseassociated modules. Moreover, total 19 common DEGs (including BCL9L, GMPS, HES1, PER2 and TSPAN33) associated with both OLP and OSCC were identified. It was clear that some novel genes might function in the malignant transformation of OLP.

$B C L 6$, which mediates B cell differentiation and inflammation, is implicated in malignant transformation via inhibiting differentiation and promoting proliferation (39). Previous study declares that BCL2 suppresses the apoptosis of immune process-associated lymphocytes and the upregulated BCL2-associated X protein (Bax) has correlation with the apoptosis of epithelial cells in patients with OLP (40). The expression alterations of BCL2, Bax, proliferating cell nuclear antigen (PCNA) and p53 proteins are observed in OLP and epithelial dysplasia, indicating the malignant potential in both lesions $(41,42)$. As a nuclear Wnt pathway component, $B C L 9$ is important for tumor progression and may be used for therapeutic intervention in several Wnt signaling-associated malignancies (43). These indicated that BCL9L might be associated with the malignant potential of OLP.

Cyclic guanosine monophosphate $(c G M P)$ plays a critical role in cell differentiation, proliferation and apoptosis, and functions in inflammation, angiogenesis and synaptic plasticity (44). Through the nitric oxide/cGMP/protein kinase G/KATP intracellular signaling pathway, inflammatory hypernociception can be inhibited by the peripheral activation of A1 adenosine receptors (A1Rs) (45). The Notch and Toll-like receptor (TLR) signaling pathways have been demonstrated to cooperately activate the expression of Notch target genes (such as Hairy/E(spl)-related with YRPW motif, HEY; and HES1) and promote the production of TLR-triggered cytokines 

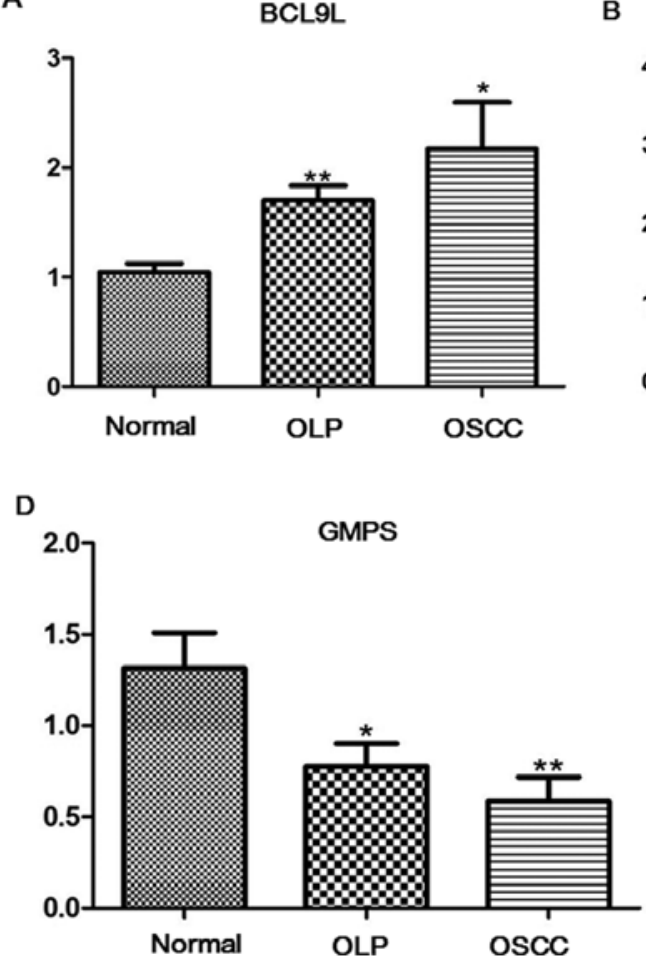

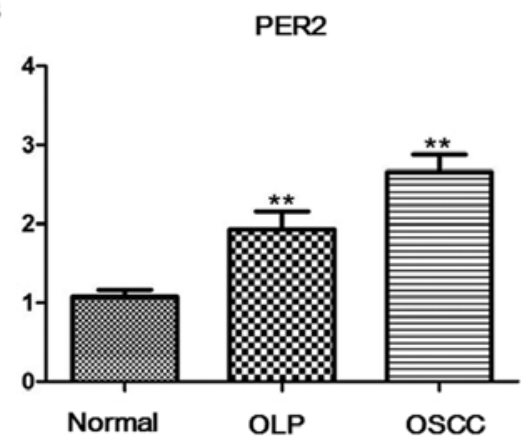

C

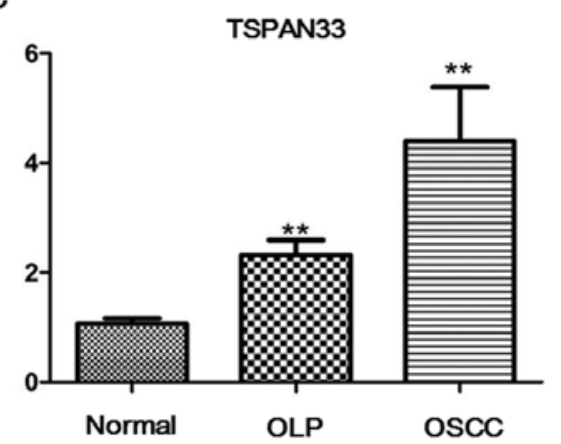

$\mathrm{E}$

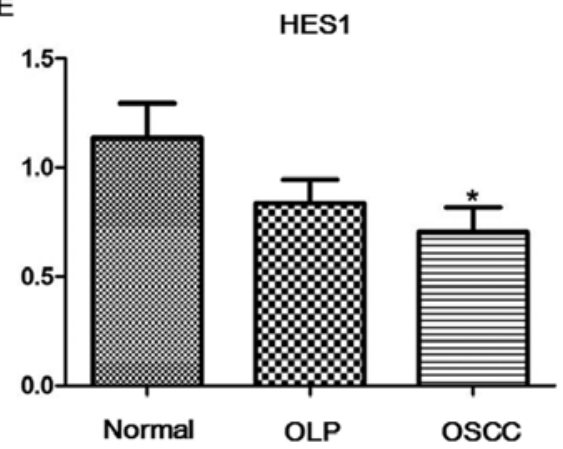

Figure 6. The expression levels of BCL9L (A), PER2 (B), TSPAN33 (C), GMPS (D) and HES1 (E) in OLP, OSCC and normal samples. "P<0.05; ${ }^{* *} \mathrm{P}<0.01$.

(such as $I L-6, I L-12$ and $T N F-\alpha$ ) (46). Several studies have suggested that the Notch signaling is related to inflammatory disorders $(47,48)$. HESI may be implicated in OSCC progression and cancer stem-like cell (CSC) phenotype in vivo, in addition, the Notch-HES1 pathway activated by inflammatory cytokine exposure may promote the phenotype in OSCC (49). Therefore, GMPS and HES1 might be involved in squamous tumorigenesis in OLP.

The mRNA and protein expression of PERl was significantly decreased in OSCC, which may be essential in the occurrence, development and invasion of OSCC (50). The tumor suppressor gene PERI acts in the development and progression of OSCC, and may be used as a molecular target for the treatment of the disease (51). Previous study reported that PER2 is important for regulating natural killer $(\mathrm{NK})$ cell function, which shows the direct link between innate immune responses and the circadian clock system (52). Using de novo mouse cancer models, Hemler et al found that select tetraspanin proteins play important roles in tumor initiation, metastasis and promotion (53). TSPAN33 shows a restricted expression pattern in activated $B$ cells, and may serve as a therapeutic target or diagnostic biomarker for B cell lymphomas or autoimmune diseases (54). The above suggest that PER2 and TSPAN33 might function in the malignant transformation of OLP to OSCC. In the co-expression networks, $B C L 9 L$, HES1, PER2 and TSPAN33 had interactions (such as BCL9LTSPAN33, BCL9L-PER2, and HES1-PER2), indicating that PER2 and TSPAN33 might also function in OLP via interacting with other genes. qRT-PCR analysis showed that BCL9L, PER2 and TSPAN33 were significantly upregulated in both OLP and OSCC samples. Whereas, GMP in both OLP and OSCC samples, and HESI in OSCC samples were significantly downregulated. The results of qRT-PCR were consistent with those of bioinformatics analysis, confirming the roles of BCL9L, GMPS, HES1, PER2 and TSPAN33 in the malignant transformation of OLP to OSCC.

In conclusion, 223 and 548 DEGs, respective, were identified in OLP vs. normal and OSCC vs. normal comparison groups through bioinformatics analysis. Furthermore, BCL9L, GMPS, HES1, PER2 and TSPAN33 acted in the malignant transformation of OLP to OSCC. However, these findings need to be further confirmed.

\section{Acknowledgements}

The present study was supported by the Medical Guiding Project, Science and Technology Commission of Shanghai Municipality, China (grant no. 134119a8700), the National Natural Science Foundation of China (grant no. 81470736), and The Shanghai Natural Science Foundation (grant no. 13ZR1405300).

\section{References}

1. van der Waal I: Potentially malignant disorders of the oral and oropharyngeal mucosa; terminology, classification and present concepts of management. Oral Oncol 45: 317-323, 2009.

2. Warnakulasuriya S, Johnson NW and van der Waal I: Nomenclature and classification of potentially malignant disorders of the oral mucosa. J Oral Pathol Med 36: 575-580, 2007.

3. Shen Z-Y, Liu W, Feng J-Q, Zhou H-W and Zhou Z-T: Squamous cell carcinoma development in previously diagnosed oral lichen planus: De novo or transformation? Oral Surg Oral Med Oral Pathol Oral Radiol Endod 112: 592-596, 2011.

4. Au J, Patel D and Campbell JH: Oral lichen planus. Oral Maxillofac Surg Clin North Am 25: 93-100, vii, 2013.

5. Gillison ML: Current topics in the epidemiology of oral cavity and oropharyngeal cancers. Head Neck 29: 779-792, 2007. 
6. Edwards BK, Brown ML, Wingo PA, Howe HL, Ward E, Ries LA, Schrag D, Jamison PM, Jemal A, Wu XC, et al: Annual report to the nation on the status of cancer, 1975-2002, featuring population-based trends in cancer treatment. J Natl Cancer Inst 97: 1407-1427, 2005

7. Choi S and Myers JN: Molecular pathogenesis of oral squamous cell carcinoma: Implications for therapy. J Dent Res 87: 14-32, 2008.

8. Patel SC, Carpenter WR, Tyree S, Couch ME, Weissler M, Hackman T, Hayes DN, Shores C and Chera BS: Increasing incidence of oral tongue squamous cell carcinoma in young white women, age 18 to 44 years. J Clin Oncol 29: 1488-1494, 2011.

9. Mollaoglu N: Oral lichen planus: A review. Br J Oral Maxillofac Surg 38: 370-377, 2000.

10. Mignogna MD, Lo Russo L, Fedele S, Ruoppo E, Califano L and Lo Muzio L: Clinical behaviour of malignant transforming oral lichen planus. Eur J Surg Oncol 28: 838-843, 2002.

11. Chen Y, Zhang W, Geng N, Tian K and Jack Windsor L: MMPs, TIMP-2, and TGF- $\beta 1$ in the cancerization of oral lichen planus. Head Neck 30: 1237-1245, 2008.

12. Mazzarella N, Femiano F, Gombos F, De Rosa A and Giuliano M: Matrix metalloproteinase gene expression in oral lichen planus: Erosive vs. reticular forms. J Eur Acad Dermatol Venereol 20: 953-957, 2006.

13. Shi P, Liu W, Zhou Z-T, He Q-B and Jiang W-W: Podoplanin and ABCG2: Malignant transformation risk markers for oral lichen planus. Cancer Epidemiol Biomarkers Prev 19: 844-849, 2010.

14. Rhodus NL, Cheng B, Myers S, Miller L, Ho V and Ondrey F: The feasibility of monitoring NF-kappaB associated cytokines: TNF- $\alpha$, IL-1 $\alpha$, IL-6, and IL-8 in whole saliva for the malignant transformation of oral lichen planus. Mol Carcinog 44: 77-82, 2005.

15. Zhang Y, Lin M, Zhang S, Wang Z, Jiang L, Shen J, Bai J, Gao F, Zhou M and Chen Q: NF-kappaB-dependent cytokines in saliva and serum from patients with oral lichen planus: A study in an ethnic Chinese population. Cytokine 41: 144-149, 2008

16. Poomsawat S, Buajeeb W, Khovidhunkit SO and Punyasingh J: Overexpression of cdk4 and p16 in oral lichen planus supports the concept of premalignancy. J Oral Pathol Med 40: 294-299, 2011.

17. Anders S, McCarthy DJ, Chen Y, Okoniewski M, Smyth GK, Huber W and Robinson MD: Count-based differential expression analysis of RNA sequencing data using R and Bioconductor. Nat Protoc 8: 1765-1786, 2013.

18. Patel RK and Jain M: NGS QC Toolkit: A toolkit for quality control of next generation sequencing data. PLoS One 7: e30619, 2012.

19. Kim D, Pertea G, Trapnell C, Pimentel H, Kelley R and Salzberg SL: TopHat2: Accurate alignment of transcriptomes in the presence of insertions, deletions and gene fusions. Genome Biol 14: R36, 2013.

20. Smyth GK: Limma: linear models for microarray data In: Bioinformatics and Computational Biology Solutions Using $\mathrm{R}$ and Bioconductor. Springer, Verlag New York Inc., NY, pp397-420, 2005.

21. Ritchie ME, Phipson B, Wu D, Hu Y, Law CW, Shi W and Smyth GK: limma powers differential expression analyses for RNA-sequencing and microarray studies. Nucleic Acids Res 43: e47, 2015

22. Deza MM and Deza E: Encyclopedia of distances. Springer, 2009. doi: 10.1007/978-3-642-00234-2.

23. Kolde R and Kolde MR: Package 'pheatmap'. 2015. http://cran.rproject.org/web/packages/pheatmap/index.html.

24. Szekely GJ and Rizzo ML: Hierarchical clustering via joint between-within distances: Extending Ward's minimum variance method. J Classif 22: 151-183, 2005.

25. Press W, Teukolsky S, Vetterling W and Flannery B: Section 16.4. Hierarchical clustering by phylogenetic trees. In: Numerical Recipes: The Art of Scientific Computing. Cambridge University Press, New York, pp868-881, 2007.

26. Oldham MC, Konopka G, Iwamoto K, Langfelder P, Kato T, Horvath S and Geschwind DH: Functional organization of the transcriptome in human brain. Nat Neurosci 11: 1271-1282, 2008

27. Liao Q, Liu C, Yuan X, Kang S, Miao R, Xiao H, Zhao G, Luo H, $\mathrm{Bu} \mathrm{D}$, Zhao $\mathrm{H}$, et al: Large-scale prediction of long non-coding RNA functions in a coding-non-coding gene co-expression network. Nucleic Acids Res 39: 3864-3878, 2011.

28. Langfelder P, Zhang B and Horvath S: Defining clusters from a hierarchical cluster tree: The Dynamic Tree Cut package for R. Bioinformatics 24: 719-720, 2008.
29. Dong $\mathbf{J}$ and Horvath S: Understanding network concepts in modules. BMC Syst Biol 1: 24, 2007.

30. Smoot ME, Ono K, Ruscheinski J, Wang P-L and Ideker T: Cytoscape 2.8: New features for data integration and network visualization. Bioinformatics 27: 431-432, 2011.

31. Falcon $\mathrm{S}$ and Gentleman R: Using GOstats to test gene lists for GO term association. Bioinformatics 23: 257-258, 2007

32. Tweedie S, Ashburner M, Falls K, Leyland P, McQuilton P, Marygold S, Millburn G, Osumi-Sutherland D, Schroeder A, Seal R, et al; FlyBase Consortium: FlyBase: Enhancing Drosophila gene ontology annotations. Nucleic Acids Res 37: D555-D559, 2009.

33. Arocho A, Chen B, Ladanyi M and Pan Q: Validation of the 2-DeltaDeltaCt calculation as an alternate method of data analysis for quantitative PCR of BCR-ABL P210 transcripts. Diagn Mol Pathol 15: 56-61, 2006.

34. Mignogna MD, Fedele S, Lo Russo L, Lo Muzio L and Bucci E: Immune activation and chronic inflammation as the cause of malignancy in oral lichen planus: Is there any evidence? Oral Oncol 40: 120-130, 2004

35. Conway C, Graham JL, Chengot P, Daly C, Chalkley R, Ross L, Droop A, Rabbitts P and Stead LF: Elucidating drivers of oral epithelial dysplasia formation and malignant transformation to cancer using RNAseq. Oncotarget 6: 40186-40201, 2015.

36. Jakhesara SJ, Koringa PG, Bhatt VD, Shah TM, Vangipuram S, Shah S and Joshi CG: RNA-Seq reveals differentially expressed isoforms and novel splice variants in buccal mucosal cancer. Gene 516: 24-32, 2013.

37. Tang X-H, Urvalek AM, Osei-Sarfo K, Zhang T, Scognamiglio T and Gudas LJ: Gene expression profiling signatures for the diagnosis and prevention of oral cavity carcinogenesis-genomewide analysis using RNA-seq technology. Oncotarget 6: 24424-24435, 2015.

38. Somoza-Martín JM, García-García A, Barros-Angueira F, Otero-Rey E, Torres-Español M, Gándara-Vila P, ReboirasLópez MD, Blanco-Carrión A and Gándara-Rey JM: Gene expression profile in oral squamous cell carcinoma: A pilot study. J Oral Maxillofac Surg 63: 786-792, 2005.

39. Shaffer AL, Yu X, He Y, Boldrick J, Chan EP and Staudt LM: BCL-6 represses genes that function in lymphocyte differentiation, inflammation, and cell cycle control. Immunity 13: 199-212, 2000

40. Fan Y, Zhan Z, Peng T, Song XL and Feng ZQ: The expression of apoptosis-associated proteins Bcl-2, Bax in oral leukoplakia and lichen planus. Shanghai Kou Qiang Yi Xue 13: 497-501, 2004 (In Chinese).

41. Hadzi-Mihailovic M, Raybaud H, Monteil R, Cakic S, Djuric M and Jankovic L: Bcl-2 expression and its possible influence on malignant transformation of oral lichen planus. J BUON 15: 362-368, 2009 .

42. Sousa FA, Paradella TC, Carvalho YR and Rosa LE Immunohistochemical expression of PCNA, p53, bax and bcl-2 in oral lichen planus and epithelial dysplasia. J Oral Sci 51: 117-121, 2009.

43. Mani M, Carrasco DE, Zhang Y, Takada K, Gatt ME, Dutta-Simmons J, Ikeda H, Diaz-Griffero F, Pena-Cruz V, Bertagnolli M, et al: BCL9 promotes tumor progression by conferring enhanced proliferative, metastatic, and angiogenic properties to cancer cells. Cancer Res 69: 7577-7586, 2009.

44. Pilz RB and Broderick KE: Role of cyclic GMP in gene regulation. Front Biosci 10: 1239-1268, 2005.

45. Lima FO, Souza GR, Verri WA Jr, Parada CA, Ferreira SH, Cunha FQ and Cunha TM: Direct blockade of inflammatory hypernociception by peripheral A1 adenosine receptors: Involvement of the NO/cGMP/PKG/KATP signaling pathway. Pain 151: 506-515, 2010.

46. Hu X, Chung AY, Wu I, Foldi J, Chen J, Ji JD, Tateya T, Kang YJ, Han J, Gessler M, et al: Integrated regulation of Toll-like receptor responses by Notch and interferon- $\gamma$ pathways. Immunity 29 : 691-703, 2008

47. Okamoto M, Takeda K, Joetham A, Ohnishi H, Matsuda H, Swasey CH, Swanson BJ, Yasutomo K, Dakhama A and Gelfand EW: Essential role of Notch signaling in effector memory $\mathrm{CD}^{+} \mathrm{T}$ cell-mediated airway hyperresponsiveness and inflammation. J Exp Med 205: 1087-1097, 2008.

48. Niranjan T, Bielesz B, Gruenwald A, Ponda MP, Kopp JB, Thomas DB and Susztak K: The Notch pathway in podocytes plays a role in the development of glomerular disease. Nat Med 14: $290-298,2008$. 
49. Lee SH, Hong HS, Liu ZX, Kim RH, Kang MK, Park NH and Shin KH: TNFo enhances cancer stem cell-like phenotype via Notch-Hes1 activation in oral squamous cell carcinoma cells. Biochem Biophys Res Commun 424: 58-64, 2012.

50. Chen R, Yang K, Zhao N-B, Zhao D, Chen D, Zhao CR and Tang H: Abnormal expression of PER1 circadian-clock gene in oral squamous cell carcinoma. Onco Targets Ther 5: 403-407, 2012.

51. Fu X-J, Li H-X, Yang K, Chen D and Tang H: The important tumor suppressor role of PER1 in regulating the cyclin-CDKCKI network in SCC15 human oral squamous cell carcinoma cells. Onco Targets Ther 9: 2237-2245, 2016.
52. Liu J, Malkani G, Shi X, Meyer M, Cunningham-Runddles S, Ma X and Sun ZS: The circadian clock Period 2 gene regulates gamma interferon production of NK cells in host response to lipopolysaccharide-induced endotoxic shock. Infect Immun 74: 4750-4756, 2006.

53. Hemler ME: Tetraspanin proteins promote multiple cancer stages. Nat Rev Cancer 14: 49-60, 2014.

54. Luu VP, Hevezi P, Vences-Catalan F, Maravillas-Montero JL, White CA, Casali P, Llorente L, Jakez-Ocampo J, Lima G, Vilches-Cisneros N, et al: TSPAN33 is a novel marker of activated and malignant B cells. Clin Immunol 149: 388-399, 2013. 